\title{
MHD Convective Flow of non-Newtonian Fluid Through Porous Medium over an Oscillating Porous Plate with Suction
}

\author{
Dhiman Bose \\ Department of Applied Mathematics, University of \\ Calcutta \\ 92 A.P.C Road, Kolkata-700009
}

\author{
Uma Basu \\ Department of Applied Mathematics, University of \\ Calcutta \\ 92 A.P.C. Road, Kolkata-700009
}

\begin{abstract}
This paper investigates the convective flow of an incompressible viscous electrically conducting second grade fluid through a porous medium occupying a semi-infinite region over an oscillating porous plate in presence of a transverse magnetic field with suction. The field equations for the velocity and temperature fields are solved analytically and the expressions for the velocity field, temperature field, skin friction and heat flux are obtained. The effects of permeability parameter, magnetic parameter/Hartmann number, Prandtl number, heat source parameter and suction parameter on the velocity field $u$ are illustrated graphically. The influence of suction parameter, Prandtl number and heat source parameter $S$ on temperature field is analyzed with the help of figures. Furthermore the effects of permeability parameter and magnetic parameter on skin friction are shown diagrammatically.
\end{abstract}

\section{Keywords}

Convective; Oscillating; Transverse Magnetic field; Suction; Porous Medium; Second Grade Fluid

\section{INTRODUCTION}

The study related to free convective flow in presence of heat source has drawn considerable attention of many researchers during last few decades because of its wide application in astrophysical sciences, cosmical studies etc. These types of flows play vital role in chemical engineering, aerospace technology etc. Unsteady oscillating flows have applications in many fields such as paper industry and many other technological fields.

Asghar et al. [1] have studied the flow of a non-Newtonian fluid induced due to the oscillations of a porous plate. Choudhury and Das [2] investigated visco-elastic MHD free convective flow through porous media in presence of radiation and chemical reaction with heat and mass transfer. Deka et al. [3] have discussed free convection effects on MHD flow past an infinite vertical oscillating plate with constant heat flux. Das et al. [4] have investigated mass transfer effects on free convective MHD flow of a viscous fluid bounded by an oscillating porous plate in the slip flow regime with heat source. A study has been done by Hayat et al. [5] on the flow of a visco-elastic fluid on an oscillating plate. Manna et al. [6] have discussed effects of radiation on unsteady MHD free convective flow past an oscillating vertical porous plate embedded in a porous medium with oscillatory heat flux. Shen et al. [7] investigated RayleighStokes problem for a heated generalized second grade fluid with fractional derivative model. Singh and Gupta [8] have studied MHD free convective flow of viscous fluid through a porous medium bounded by an oscillating porous plate in slip flow regime with mass transfer.

In present problem a convective flow of an incompressible non-Newtonian fluid through a porous medium over an oscillating porous plate in presence of transverse magnetic field has been considered. It is observed that an increase in the parameter of permeability $K_{p}$ leads to an increase in the velocity field $u$. The velocity field $u$ decreases with an increase in magnetic field parameter $M$, Prandtl number $\mathrm{P}_{\mathrm{r}}$, heat source parameter $S$ and suction parameter $v_{0}$ as shown in figures. It can be shown in the figure that the temperature field $\mathrm{T}$ decreases with an increase in suction parameter $\mathrm{v}_{0}$, Prandtl number $\mathrm{P}_{\mathrm{r}}$, heat source parameter $\mathrm{S}$ separately. Further it is observed that the period of oscillation of the stress curves increases with an increase in the parameter of permeability $\mathrm{K}_{\mathrm{p}}$.

\section{ANALYSIS OF THE PROBLEM}

Let us consider the convective flow of an incompressible viscous electrically conducting second grade fluid through a porous medium occupying a semi-infinite region over an oscillating porous plate in presence of transverse magnetic field $\mathrm{B}_{0}$. Let $\mathrm{u}$ and $\mathrm{v}$ are the velocity components in the $\mathrm{x}$ - and $\mathrm{y}$-directions respectively and the direction of $\mathrm{x}$ - and $\mathrm{y}$ axes are taken respectively along and normal to the plate. Since the plate is infinite dimensional in $\mathrm{x}$ and $\mathrm{z}$-directions, all the physical quantities are functions of $\mathrm{y}$ and $\mathrm{t}$ only. The Reynolds number is assumed to be very small and the induced magnetic field due to the flow is neglected with respect to the applied magnetic field. The pressure is assumed to be constant in the flow field. If $\mathrm{v}_{0}$ be the suction/injection velocity at the plate then the equation of continuity is given by $\frac{\partial \mathrm{v}}{\partial \mathrm{y}}=0$

Under the condition $\mathrm{y}=0, \mathrm{v}=-\mathrm{v}_{0}$ then the governing boundary layer equation is given by

$\frac{\partial \mathrm{u}}{\partial \mathrm{t}}-\mathrm{v}_{0} \frac{\partial \mathrm{u}}{\partial \mathrm{y}}=\left(v+\alpha \frac{\partial}{\partial \mathrm{t}}\right) \frac{\partial^{2} \mathrm{u}}{\partial \mathrm{y}^{2}}+\mathrm{g} \beta\left(\mathrm{T}-\mathrm{T}_{\infty}\right)-\frac{v}{\mathrm{~K}_{0}} \mathrm{u}-$

$\frac{\sigma \mathrm{B}_{0}^{2} \mathrm{u}}{\rho}$

$\frac{\partial \mathrm{T}}{\partial \mathrm{t}}-\mathrm{v}_{0} \frac{\partial \mathrm{T}}{\partial \mathrm{y}}=$

$\kappa\left(1+\alpha \frac{\partial}{\partial \mathrm{t}}\right) \frac{\partial^{2} \mathrm{~T}}{\partial \mathrm{y}^{2}}-\mathrm{S}\left(\mathrm{T}-\mathrm{T}_{\infty}\right)$

Where $\alpha$ is normal stress moduli, $\kappa$ is thermal diffusivity, $v$ is kinematic viscosity, $g$ is acceleration due to gravity, $\beta$ is the volumetric coefficient of expansion for heat transfer, $\mathrm{K}_{0}$ is permeability of the porous medium, $\rho$ is the density, $\sigma$ is electrical conductivity of the fluid, $\mathrm{S}$ is the heat source 
parameter, $\mathrm{T}$ is the temperature, $\mathrm{T}_{\infty}$ is the temperature of the fluid far away from the plate.

The boundary conditions are given by

$$
\begin{aligned}
\mathrm{u}=\mathrm{U}_{0} \mathrm{e}^{\mathrm{i} \omega \mathrm{t}}, \mathrm{T}=\mathrm{T}_{\mathrm{w}} \text { at } \mathrm{y}=0 \\
\mathrm{u} \rightarrow 0, \mathrm{~T} \rightarrow \mathrm{T}_{\infty} \text { as } \mathrm{y} \rightarrow \infty
\end{aligned}
$$

The non-dimensional variables are introduced as follows

$$
\begin{gathered}
\mathrm{y}^{\prime}=\mathrm{U}_{0} \frac{\mathrm{y}}{v}, \mathrm{u}^{\prime}=\frac{\mathrm{u}}{\mathrm{U}_{0}}, \mathrm{~T}^{\prime}=\frac{\mathrm{T}-\mathrm{T}_{\infty}}{\mathrm{T}_{\mathrm{w}}-\mathrm{T}_{\infty}}, \mathrm{t}^{\prime}=\mathrm{U}_{0}^{2} \frac{\mathrm{t}}{v}, \\
\mathrm{v}_{0}^{\prime}=\frac{\mathrm{v}_{0}}{\mathrm{U}_{0}}, \omega^{\prime}=\frac{v \omega}{\mathrm{U}_{0}^{2}}, \mathrm{~S}^{\prime}=\frac{v \mathrm{~S}}{\mathrm{U}_{0}^{2}}, \mathrm{M}=\frac{\mathrm{B}_{0}}{\mathrm{U}_{0}}\left(\frac{v \sigma}{\rho}\right)^{\frac{1}{2}}, \alpha^{\prime}=\alpha \frac{\mathrm{U}_{0}^{2}}{v^{2}}, \\
\mathrm{P}_{\mathrm{r}}=\frac{v}{\mathrm{k}}, \mathrm{K}_{\mathrm{p}}=\frac{\mathrm{K}_{0} \mathrm{U}_{0}^{2}}{v^{2}}, \mathrm{G}_{\mathrm{r}}=v g \beta \frac{\mathrm{T}_{\mathrm{w}}-\mathrm{T}_{\infty}}{\mathrm{U}_{0}^{3}}
\end{gathered}
$$

Where $\mathrm{M}$ is Hartmann number/magnetic field parameter, $\mathrm{P}_{\mathrm{r}}$ is Prandtl number, $K_{p}$ is the permeability parameter, $G_{r}$ is Grashof number for heat transfer.

The governing boundary layer equations (2) and (3) in terms of non-dimensional variables becomes (Dropping ' sign for convenience)

$$
\begin{aligned}
& \frac{\partial u}{\partial t}-v_{0} \frac{\partial u}{\partial y}=\left(1+\alpha \frac{\partial}{\partial t}\right) \frac{\partial^{2} u}{\partial y^{2}}+G_{r} T-\left(\frac{1}{K_{p}}+M^{2}\right) u \\
& \frac{\partial T}{\partial t}-v_{0} \frac{\partial T}{\partial y}=\frac{1}{P_{r}}\left(1+\alpha \frac{\partial}{\partial t}\right) \frac{\partial^{2} T}{\partial y^{2}}-S T
\end{aligned}
$$

Then the non-dimensional boundary conditions are given by

$$
\begin{aligned}
\mathrm{u}= & \mathrm{e}^{\mathrm{i} \omega \mathrm{t}}, \mathrm{T}=1 \text { at } \mathrm{y}=0 \\
\mathrm{u} & \rightarrow 0, \mathrm{~T} \rightarrow 0 \text { as } \mathrm{y} \rightarrow \infty
\end{aligned}
$$

\section{SOLUTION OF THE PROBLEM}

For solving the equations (5) and (6) the velocity and temperature fields are assumed to be of the following forms

$$
u=u_{0}+u_{1} e^{i \omega t}
$$

$$
\text { And } \mathrm{T}=\mathrm{T}_{0}+\mathrm{T}_{1} \mathrm{e}^{\mathrm{i} \omega \mathrm{t}}
$$

Where $u_{i}, T_{i}(i=0,1)$ are functions of $y$ only.

Substituting the expressions for $\mathrm{u}$ and $\mathrm{T}$ from (8) and (9) respectively in equations (5) and (6) and separating harmonic and non-harmonic terms the following equations are obtained

$$
\begin{gathered}
\frac{d^{2} u_{0}}{d y^{2}}+v_{0} \frac{d u_{0}}{d y}-\left(\frac{1}{K_{p}}+M^{2}\right) u_{0}=-G_{r} T_{0} \\
\frac{d^{2} u_{1}}{d y^{2}}+\frac{v_{0}}{1+i \alpha \omega} \frac{d u_{1}}{d y}-\frac{\left(\frac{1}{K_{p}}+M^{2}+i \omega\right)}{1+i \alpha \omega} u_{1} \\
=\frac{-G_{r} T_{1}}{1+i \alpha \omega} \\
\frac{d^{2} T_{0}}{d y^{2}}+P_{r} v_{0} \frac{d T_{0}}{d y}+P_{r} S T_{0}=0 \\
\frac{d^{2} T_{1}}{d y^{2}}+\frac{P_{r} v_{0}}{1+i \alpha \omega} \frac{d T_{1}}{d y}+\frac{P_{r}(S-i \omega)}{1+i \alpha \omega} T_{1}=0
\end{gathered}
$$

The corresponding boundary conditions are given by

$$
\begin{aligned}
& \mathrm{u}_{0}=0, \mathrm{u}_{1}=1 \text { at } \mathrm{y}=0 \\
& \mathrm{u}_{0}=0, \mathrm{u}_{1}=0 \text { at } \mathrm{y} \rightarrow \infty
\end{aligned}
$$

$\mathrm{T}_{0}=\mathrm{T}_{1}=0$ at $\mathrm{y} \rightarrow \infty$

The solutions of the equations (10) - (13) under the boundary conditions (14) to (17) are obtained as

$$
\begin{array}{r}
u_{0}=\frac{G_{r}}{m_{1}^{2}+v_{0} m_{1}-\left(\frac{1}{K_{p}}+M^{2}\right)}\left[e^{\frac{-v_{0}-\sqrt{v_{0}^{2}+4\left(\frac{1}{K_{p}}+M^{2}\right)}}{2} y}-e^{m_{1} y}\right] \\
u_{1}=e^{\left\{\frac{v_{0}}{1+i \alpha \omega}-\sqrt{\left.\left(\frac{v_{0}}{1+i \alpha \omega}\right)^{2}+\frac{4\left(\frac{1}{K_{p}}+M^{2}+i \omega\right)}{1+i \alpha \omega}\right)}\right\} y} \\
T_{0}=e^{m_{1} y} \\
T_{1}=0
\end{array}
$$

Therefore the equations (8) and (9) yield the velocity and temperature fields as

$$
\begin{gathered}
u=\frac{G_{r}}{m_{1}^{2}+v_{0} m_{1}-\left(\frac{1}{K_{p}}+M^{2}\right)}\left[e^{\frac{-v_{0}-\sqrt{v_{0}^{2}+4\left(\frac{1}{K_{p}}+M^{2}\right)}}{2} y}-e^{m_{1} y}\right] \\
+e^{\frac{-v_{0}-U-\alpha \omega V}{1+\alpha^{2} \omega^{2} y} \cos \left(\omega t+\frac{\alpha \omega v_{0}+\alpha \omega U-V}{1+\alpha^{2} \omega^{2}} y\right)} \\
T=e^{m_{1} y}
\end{gathered}
$$

Where $U=\frac{1}{\sqrt{2}} \sqrt{\sqrt{P^{2}+Q^{2}}+P}, V=\frac{1}{\sqrt{2}} \sqrt{\sqrt{P^{2}+Q^{2}}-P}$, $P=v_{0}^{2}+4\left(\frac{1}{K_{p}}+M^{2}-\alpha \omega^{2}\right), Q=4\left(\omega+\frac{\alpha \omega}{K_{p}}+M^{2} \alpha \omega\right)$

The skin friction at the plate is given by

$$
\begin{aligned}
& \tau=\left(\frac{\partial u}{\partial y}\right)_{y=0}=\frac{G_{r}}{m_{1}^{2}+v_{0} m_{1}-\left(\frac{1}{K_{p}}+M^{2}\right)}\left[\frac{-v_{0}-\sqrt{v_{0}^{2}+4\left(\frac{1}{K_{p}}+M^{2}\right)}}{2}-m_{1}\right] \\
& -\frac{v_{0}+U+\alpha \omega V}{1+\alpha^{2} \omega^{2}} \cos \omega t-\sin \omega t \frac{\alpha \omega v_{0}+\alpha \omega U-V}{1+\alpha^{2} \omega^{2}}
\end{aligned}
$$

The heat flux at the plate in terms of Nusselt number is given by

$$
N_{u}=\left(\frac{\partial T}{\partial y}\right)_{y=0}=m_{1}=\frac{-P_{r} v_{0}-\sqrt{P_{r}^{2} v_{0}^{2}-4 P_{r} S}}{2}
$$

\section{RESULTS AND DISCUSSION}

In present paper a study on the free convective flow of an unsteady incompressible electrically conducting second grade fluid through a porous medium occupying semi-infinite region over a porous oscillating plate in presence of transverse magnetic field with suction has been made. The constitutive equation for the stress considered here is related to second grade fluid. The expressions for velocity field, temperature field are obtained analytically by solving the field equations. The skin friction and heat flux at the plate are found out as partial derivatives of velocity and temperature fields respectively. The effects of the flow parameters $K_{p}, M, P_{r}, S, v_{0}$ and $\alpha$ on velocity field $u$ have been discussed with the help of Figures 1-5 and Figure 11 respectively. The effects of $v_{0}$ and $\mathrm{P}_{\mathrm{r}}, S$ on temperature field $T$ have been illustrated graphically with the help of Figures 6-8. Furthermore the influence of $M$ on stress field $\tau$ is presented pictorially in Figure 9. It is observed that the stress field related to the flow changes with the variation of the flow parameters.

In Figure 1 velocity field is depicted against y for different values of $K_{p}$. The figure shows that velocity field sharply

$\mathrm{T}_{0}=1, \mathrm{~T}_{1}=0$ at $\mathrm{y}=0$ 
decreases from a finite value as y increases and approaches zero. By comparing the velocity curves in the figure it is observed that a growing permeability parameter $K_{p}$ leads to accelerate the magnitude of velocity field at all points. Figure 2 shows that the increase in Hartmann number $M$ results in decrease in velocity field. This is expected, as the magnetic field has a retarding influence on the flow fields. In Figure 3 velocity field is depicted against y for different values of Prandtl number $P_{r}$. The figure shows that the velocity field decreases with increase in $P_{r}$. Figure 4 discusses the effects of heat source parameter $S$ on the velocity field $u$. From the figure it can be seen that the velocity field $u$ sharply decreases from a finite value as y increases near the boundary layer. The figure shows that increase in the parameter $S$ leads to decrease in the magnitude of the velocity field. The velocity field is depicted against y for different values of suction velocity $v_{0}$ in Figure 5. The velocity field decreases with the increase in $v_{0}$. The velocity decreases sharply from a definite velocity with increase in $\mathrm{y}$ and becomes zero finally. In Figure 6 temperature field is plotted against y for different values of suction velocity $v_{0}$. The temperature decreases sharply with the increase in y near the boundary layer and approaches zero. Temperature field $T$ decreases with the increase in suction velocity $v_{0}$ very near to the plate. Temperature field is depicted against y for different values of $P_{r}$ in Figure 7. From there it can be seen that an increase in y leads to sharp decrease in $T$ and $T$ approaches zero finally. As the Prandtl number takes higher values the temperature field $T$ decreases very near to the plate. Figure 8 shows the plot of temperature field $T$ against y for different values of heat source parameter $S$. The temperature field $T$ decreases sharply with increase in y near the boundary layer and approaches zero. $T$ decreases as the heat source parameter $S$ takes higher values near the plate. The skin friction $\boldsymbol{\tau}$ is depicted against $\omega$ for different values of $M$ in Figure 9. It is evident from the figure that the stress curves are oscillatory in nature. There are points of separation at which the stress $\tau$ becomes zero. The figure shows that for the flow of non-Newtonian fluid in presence of magnetic field the amplitude of the stress curve decreases with the increase in $\omega$ as shown in Figure 9. In Figure $\mathbf{1 0}$ the velocity field is plotted against time $\mathrm{t}$ for different values of $\mathrm{y}$. The figure shows that the velocity curves are oscillatory in nature. There are points about which the magnitude of amplitude of oscillation of the velocity curve changes its direction for different values of $y$ periodically. The amplitude of oscillation of the velocity curve decreases for increasing values of $y$ on the left of that points and then on the right of that points the direction of amplitude changes periodically and as $y$ takes larger values the amplitude of oscillation of the velocity curve becomes zero. Figure 11 shows the effects of normal stress moduli $\alpha$ on the velocity field $u$. There are sharp decrease in the velocity field $u$ with the increase in $y$. It can be observed that as $\alpha$ increases the magnitude of the velocity field increases. The velocity curve for the flow of Newtonian fluid has also been plotted in the figure. It is also seen that there are negative velocities in the boundary layer and for large values of $\alpha$, there is an incipient flow reversal near the plate. In Table 1 the stress values for different values of magnetic field parameter $M$ and frequency of oscillation $\omega$ with $G_{r}=$ $1, v_{0}=1, K_{p}=10, t=0.4, P_{r}=0.5, S=5, \alpha=0.0 \quad$ are computed. From the figure it can be seen that the stress field $\tau$ oscillates with $\omega$ for fixed values of $M$ and it is the case seen in Figure 9. It is also evident from the Table that the stress field is monotonic function of $M$ for fixed value of $\omega$.

\section{FIGURES}

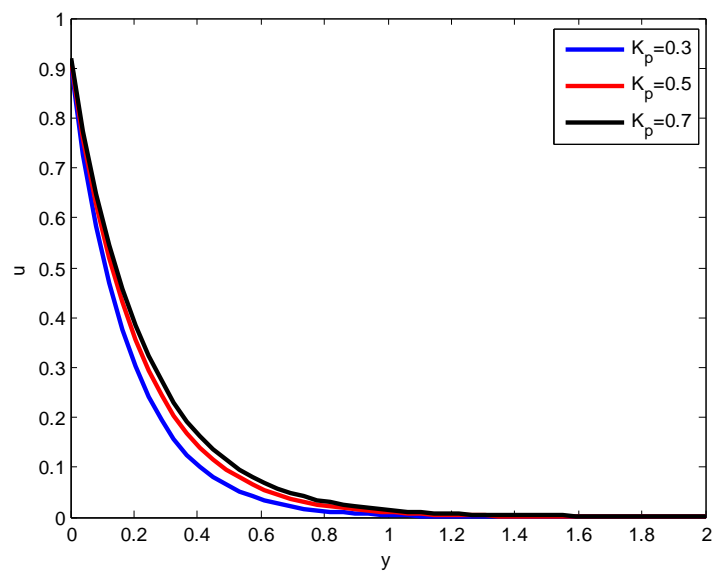

Figure 1. Velocity profile is depicted against $y$ for different values of permeability parameter $K_{p}$ with $G_{r}=1, M=1, \alpha=0.3, v_{0}=1, \omega=0.2, t=0.4, P_{r}=$ $0.3, S=0.5$

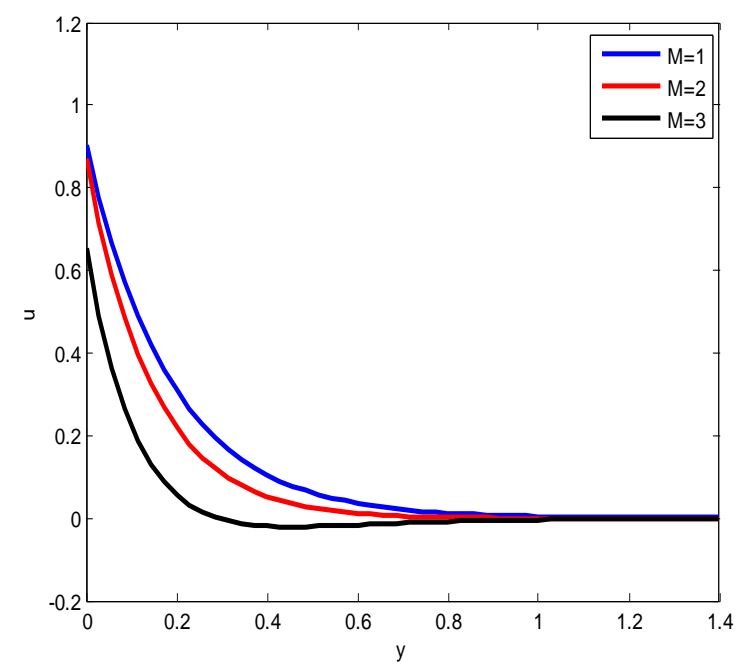

Figure 2. Velocity profile is depicted against $y$ for different values of magnetic field parameter $M$ with $G_{r}=1, v_{0}=$ $1, K_{p}=0.3, \alpha=0.3, \omega=0.2, t=0.4, P_{r}=5, S=0.5$

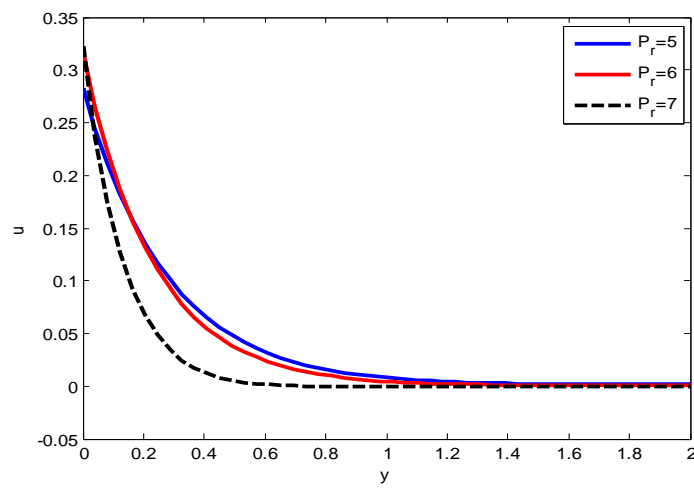

Figure 3. Velocity profile is depicted against $y$ for different values of Prandtl number $P_{r}$ with $G_{r}=1, v_{0}=1, M=$ $1, \alpha=0.3, K_{p}=0.3, \omega=0.2, t=0.4, S=0.5$ 


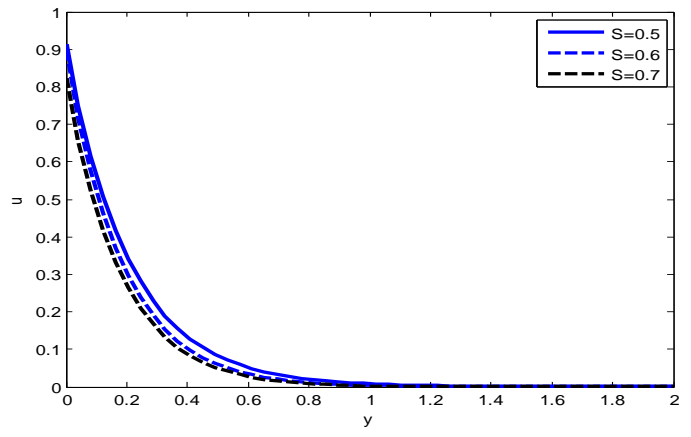

Figure 4. Velocity profile is depicted against $y$ for different values of heat source parameter $S$ with $G_{r}=1, M=1, \alpha=$ $0.3, v_{0}=1, K_{p}=0.3, \omega=0.2, t=0.4, P_{r}=5$

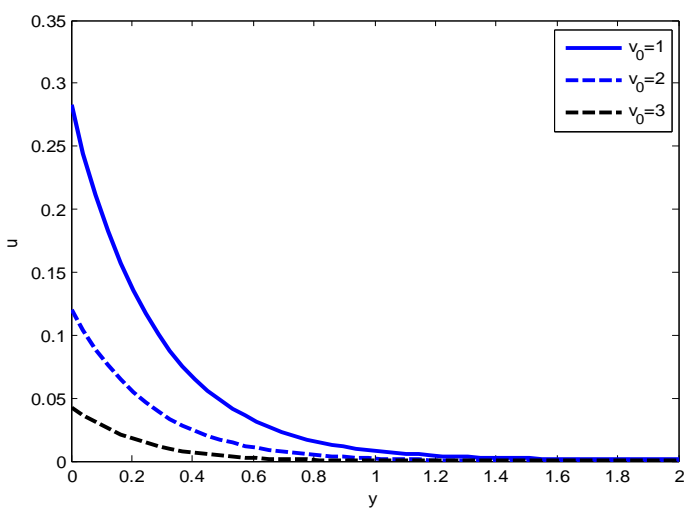

Figure 5. Velocity profile is depicted against $y$ for different values of suction velocity $v_{0}$ with $G_{r}=1, K_{p}=0.3, M=$ $1, \alpha=0.3, \omega=0.2, t=0.4, P_{r}=5, S=5.0$

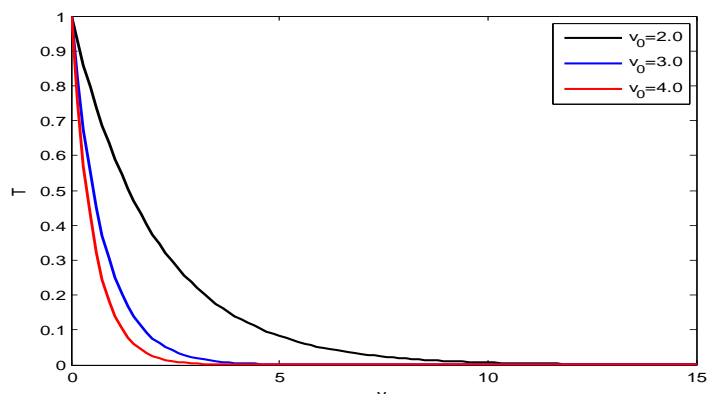

Figure 6. temperature $T$ is depicted against $y$ for different values of suction velocity $v_{0}$ with $P_{r}=0.5, S=0.5$

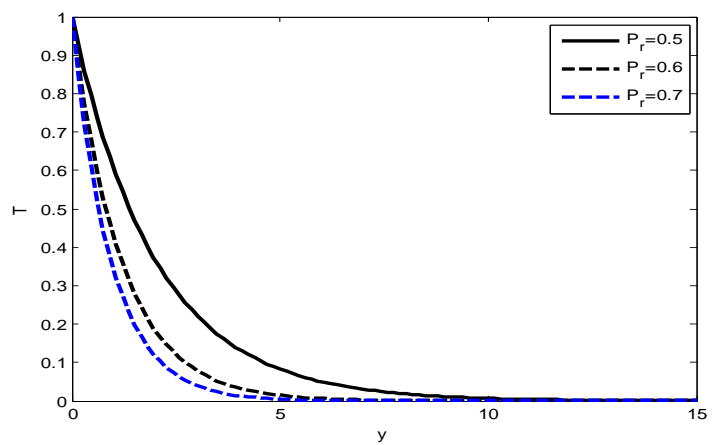

Figure 7. Temperature $T$ is depicted against $y$ for different values of Prandtl number $P_{r}$ with $v_{0}=2.0, S=0.5$

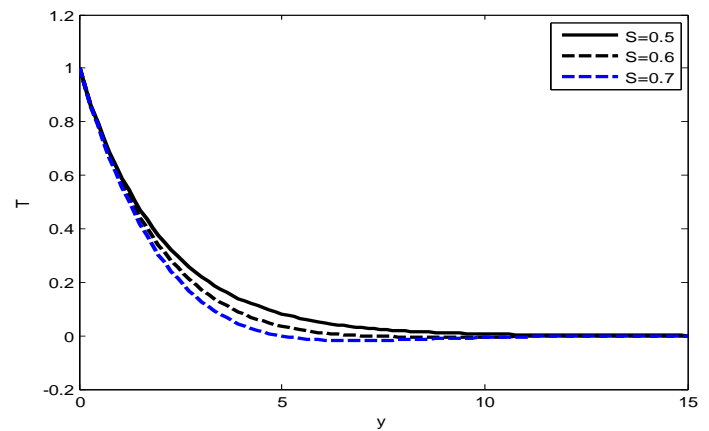

Figure 8. Temperature $T$ is depicted against $y$ for different values of heat source parameter $S$ with $P_{r}=0.5, v_{0}=2.0$

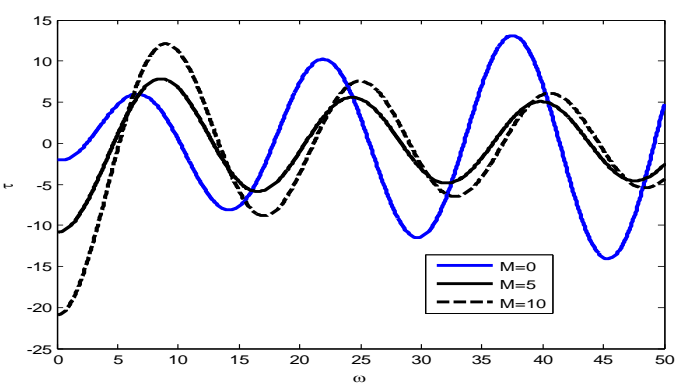

Figure 9. Skin friction $\tau$ is depicted against $\omega$ for different values of magnetic field parameter $M$ with $K_{p}=0.3, G_{r}=$ $1, t=0.4, \alpha=0.3, P_{r}=0.5, S=5, v_{0}=1.0$

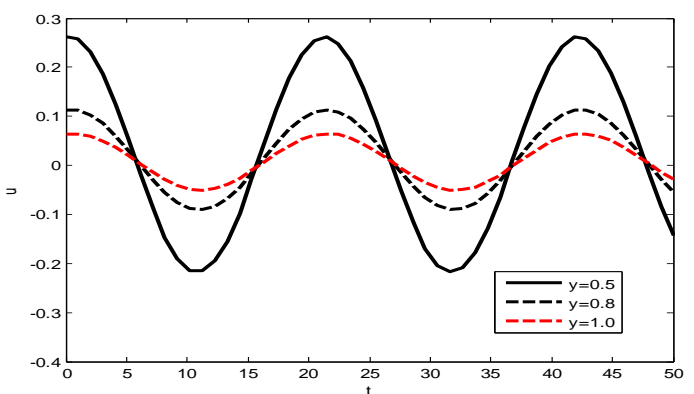

Figure 10 The velocity field is plotted against time $t$ for different

values of $y$ with $G_{r}=1.0, K_{p}=3, M=0.5, \alpha=$ $0.2, \mathrm{v}_{0}=1.0$,

$\omega=0.5, P_{r}=0.5, S=5$

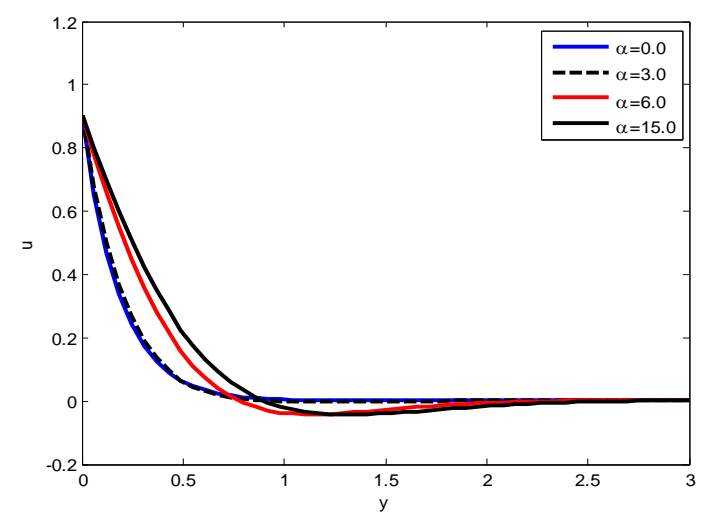

Figure11. The velocity is depicted against $y$ for different values of normal stress moduli $\alpha$ with $G_{r}=1, M=1, v_{0}=$ $1, K_{p}=0.3, \omega=0.2, t=0.4, P_{r}=5, S=0.5$ 
Table 1. Shear stress at the plate $y=0$ with $G_{r}=1, v_{0}=$ $1, K_{p}=10, t=0.4, P_{r}=0.5, S=5, \alpha=0.0$

\begin{tabular}{|c|c|c|c|c|}
\hline$\omega \backslash M$ & 0 & 5 & 10 & 15 \\
\hline 0 & -2.0495 & -10.8809 & -20.9351 & -30.9563 \\
\hline 5 & 4.6893 & 5.7143 & 9.3098 & 13.2809 \\
\hline 10 & 0.4363 & 6.0711 & 13.1109 & 19.8460 \\
\hline 15 & -7.6599 & -11.6245 & -20.5679 & -30.0157 \\
\hline 20 & 7.4103 & 5.5824 & 5.1403 & 5.9020 \\
\hline
\end{tabular}

\section{ACKNOWLEDGEMENTS}

We are grateful to the reviewers for their valuable comments to make this paper in the present format.

\section{REFERENCES}

[1] Asghar, S., Mohyuddin, M.R., Hayat, T. and Siddiqui, A.M. 2004. The flow of a non-Newtonian fluid induced due to the oscillations of a porous plate. Math. Prob. Engng., 2,133-143.

[2] Choudhury, R. and Das, S.K. 2014. Visco-elastic MHD free convective flow through porous media in presence of radiation and chemical reaction with heat and mass transfer. Journal of Applied Fluid Mechanics, 7, 603609.
[3] Deka, R.K., Das, U.N. and Soundalgekar, V.M. 1997. Free convection effects on MHD flow past an infinite vertical oscillating plate with constant heat flux. Ind. J. Math, 39, 195-202.

[4] Das, S.S., Tripathy, R.K., Sahoo, S.K. and Dash, B.K 2008. Mass transfer effects on free convective MHD flow of a viscous fluid bounded by an oscillating porous plate in the slip flow regime with heat source. J. Ultra Scientist of Phys. Sci. 20, 169-176.

[5] Hayat, T., Mohyuddin, M.R., Asghar, S., Siddiqui, A.M 2004. The flow of a visco-elastic fluid on an oscillating plate. Z. Angew. Math. Mech., 84, 65-70.

[6] Manna, S. S., Das, S. and Jana, R.N. 2012. Effects of radiation on unsteady MHD free convective flow past an oscillating vertical porous plate embedded in a porous medium with oscillatory heat flux. Advances in Applied Science Research, 3, 3722-3736.

[7] Shen, F., Tan, W., Zhao, Y. and Masuoka, T. 2006. The Rayleigh-Stokes problem for a heated generalized second grade fluid with fractional derivative model. Nonlinear Analysis: Real World Applications, 7, 1072-1080.

[8] Singh, P. and Gupta, C.B. 2005. MHD free convective flow of viscous fluid through a porous medium bounded by an oscillating porous plate in slip flow regime with mass transfer. Ind. J. Theo. Phys., 53, 111-120. 